\title{
Verdrängung von Männer- durch Frauenarbeit? Ein politisch wirkungs- mächtiges Klischee auf dem Prüfstand der Reichsstatistik. Untersucht am Beispiel der Metallindustrie, 1895-1933'
}

\author{
Von Brigitte Kassel (Berlin)
}

Ein bis heute zählebiges Klischee in der Geschichte der Frauenerwerbsarbeit ist die These von der Verdrängung der Männerarbeit durch billigere Frauenarbeit. ${ }^{2}$ Die Angst der Männer vor Verdrängung mag in männlich dominierten Erwerbsbereichen wie der Metallindustrie, die als Fallbeispiel analysiert werden soll, besonders groß gewesen sein. Unbestreitbar ist, daß im freigewerkschaftlichen Deutschen Metallarbeiter-Verband (DMV) Frauenerwerbsarbeit immer unter negativen Vorzeichen diskutiert wurde. Die quantitative Entwicklung der Frauenerwerbsarbeit wurde mißtrauisch verfolgt. Zwar akzeptierte man notgedrungen die ökonomische Notwendigkeit von Frauenerwerbsarbeit, doch die Akzeptanz hatte ihre Grenzen. Diese zeigten sich in aller Deutlichkeit bei der personellen Demobilmachung nach dem Ersten Weltkrieg. ${ }^{3}$ Angesichts hoher konjunktureller und struktureller Arbeitslosigkeit gewann schließlich die "Verdrängungsthese", die die Verantwortung für die Männerarbeitslosigkeit auf die erwerbstätigen Frauen schob, in den krisenhaften Jahren der Weimarer Republik besondere politische Brisanz. Forderungen nach Einschränkung der Frauenerwerbsarbeit und ganz besonders der Ehefrauenerwerbsarbeit waren an der Tagesordnung. Neben Berufsberatung und produktiver Erwerbslosenfürsorge ${ }^{4}$ schienen Arbeitsverbote für Frauen zu einem wesentlichen Mittel im Repertoire der Bewältigungsstrategien von Arbeitsmarktproblemen zu werden.

Die Politik mit der Frauenfrage verzichtete weitgehend auf Zahlen, durch die die Zunahme der Frauenerwerbsarbeit zum Nachteil der Männer überzeugend belegt worden wäre. Obwohl der DMV besonders in den Jahren bis zum Ersten Weltkrieg zahlreiche Erhebungen zur Situation einzelner Berufsgruppen und zur Entwicklung verschiedener Branchen durchführte, widmete er sich in seiner mehr als vierzigjährigen Geschichte lediglich in zwei Erhebungen von 1916 und 1930 ausführlich dem Thema Frauenarbeit in der Metallindustrie.

Der skeptische Blick in die Zukunft motivierte die Untersuchung von 1916. Dem DMV bereitete Sorgen, was nach Kriegsende mit den während des Krieges von Frauen besetzten Arbeitsplätzen in der Metallindustrie geschehen würde, wie durch Frauenarbeit die Arbeitsbedingungen langfristig negativ beeinflußt wurden und was nach dem Krieg mit denjenigen Arbeiterinnen geschehen sollte, die auf Erwerb angewiesen waren. Die Erhebung von 1930 entstand unter dem Eindruck einer vermeintlich erheblichen Zunahme der Frauenarbeit

1 Der Aufsatz basiert auf meiner Dissertation: Frauen in einer Männerwelt. Frauenerwerbsarbeit in der Metallindustrie und ihre Interessenvertretung durch den Deutschen Metallarbeiter-Verband (1891-1933), TU Berlin 1995 (jetzt: Bund-Verlag, Köln 1997), die aus Mitteln des Förderprogramms Frauenforschung des Berliner Senats gefördert wurde. Für Hinweise und Kritik danke ich vor allem Karin Hausen und Karin Zachmann.

2 Zu den Klischees in der historischen Forschung über Frauenarbeit: $\boldsymbol{K}$. Hausen, Wirtschaften mit der Geschlechterordnung, in: dies. (Hg.), Geschlechterhierarchie und Arbeitsteilung. Zur Geschichte ungleicher Erwerbschancen von Männern und Frauen, Göttingen 1993, S. 40-67.

3 Siehe hierzu v.a. S. Rouette, Sozialpolitik als Geschlechterpolitik. Die Regulierung der Frauenarbeit nach dem Ersten Weltkrieg, Frankfurt a.M. 1993.

4 Siehe L. Preller, Sozialpolitik in der Weimarer Republik, Düsseldorf 1978. 
infolge der Rationalisierungsmaßnahmen seit Mitte der zwanziger Jahre und der Annahme, diese hätten zu einer bedeutenden Umschichtung auf dem Arbeitsmarkt geführt. ${ }^{5}$ In beiden Fällen hatte die ablehnende Haltung der Gewerkschaft gegenüber Frauenerwerbsarbeit und die Abwehr einer befürchteten Gleichstellung von Männer- und Frauenarbeit in der Metallindustrie erheblichen Einfluß auf die Konzeption und Analyse der Erhebungen.

Bemerkenswert ist erstens, daß die Gruppe der weiblichen Arbeitskräfte völlig isoliert von der Gruppe der männlichen Arbeitskräfte untersucht wurde. Weder die Zahl der Arbeiterinnen noch deren Entlohnung wurde in Relation zur Männerarbeit betrachtet. Zweitens wurden die Ergebnisse dadurch vorprogrammiert, daß im Zentrum der Erhebungen lediglich Orte und Betriebe standen, die für die Frauenerwerbsarbeit Bedeutung hatten. Dieser Ansatz führte zu einer quantitativen Überschätzung der Frauenarbeit, die jedoch nicht thematisiert wurde. Der Blick war fixiert auf in der Tat beeindruckende Steigerungsquoten der weiblichen Beschäftigung im Verlauf des Krieges. So wurde beispielsweise festgestellt, daß die Zahl der Arbeiterinnen in der Metallindustrie Rheinland-Westfalens von August 1914 bis August 1916 um 685 Prozent gestiegen sei. ${ }^{6}$ Auch 1930 war in erster Linie der Zuschnitt der Untersuchung dafür verantwortlich, daß sogar im Bereich der Eisen- und Metallgewinnung ein Frauenanteil von nahezu 20 Prozent festgestellt wurde.' Der weitverbreiteten Furcht vor einer Verdrängung der Männer- durch Frauenarbeit wurde also reichlich Nahrung gegeben.

Zeitgenossen und Zeitgenossinnen widerlegten die Verdrängungsthese mit guten Argumenten und Zahlen, ${ }^{8}$ was die männliche Arbeiterschaft der "Männerindustrien“ und besonders ihre gewerkschaftlich organisierten Vertreter jedoch nicht anfocht. Und in der historischen Forschung verweisen Betriebsstudien zu Siemens und Bosch darauf, daß aufgrund konjunktureller Faktoren und unterschiedlicher Qualifikationsanforderungen am konkreten Arbeitsplatz Männer und Frauen auf ganz verschiedene Weise von Einstellungs- und Entlassungswellen betroffen waren.'

Ich werde im folgenden den Nachweis führen, daß auch die aggregierten Daten der Reichsstatistik die Verdrängungsthese keineswegs stützen. Dabei orientiere ich mich an

5 Die Frauenarbeit in der Metallindustrie während des Krieges. Dargestellt nach Erhebungen im Aug./September 1916 vom Vorstand des Deutschen Metallarbeiter-Verbandes, Stuttgart 1917, S. 7 f. (im folgenden: Frauenarbeit 1916). Vorstand des Deutschen Metallarbeiter-Verbandes (Hg.), Die Frauenarbeit in der Metallindustrie, Stuttgart 1930, S. VII (im folgenden: Frauenarbeit 1930). Darüber hinaus wurde 1915 von den Metallarbeiterorganisationen im Rheinland und in Westfalen eine Erhebung zur Verbreitung der Frauenarbeit vorgenommen: Vorstand des DMV ( $\mathrm{Hg}$.$) , Jahr- und Handbuch des Deutschen$ Metallarbeiter-Verbandes für das Jahr 1915, Stuttgart 1916, S. $304 \mathrm{f}$

6 Frauenarbeit 1916, S. 8 f.

7 Frauenarbeit 1930 , S. 32.

8 Siehe u.a. L. Nielsen, Die Verdrängung von Männerarbeit durch Frauenarbeit in der Industrie, Phil. Diss. Bonn 1919; H. Roehl, Die gewerbliche Bildungsfrage für erwerbstätige Frauen unter besonderer Rücksicht auf das Handwerk, in: Kultur und Fortschritt 282/83, 1910, S. 2-16; siehe auch A. Willms(-Herget), Die Entwicklung der Frauenerwerbstätigkeit im Deutschen Reich, Nürnberg 1980, S. 148 ff.; dies., Frauenarbeit. Zur Integration der Frauen in den Arbeitsmarkt, Frankfurt a.M. 1985, S. 150-167.

9 U. Stolle, Arbeiterpolitik im Betrieb. Frauen und Männer, Reformisten und Radikale, Fach- und Massenarbeiter bei Bayer, BASF, Bosch und in Solingen (1900-1933), Frankfurt a.M. 1980, S. 197 f.; Th. von Freyberg, Industrielle Rationalisierung in der Weimarer Republik. Untersucht an Beispielen aus dem Maschinenbau und der Elektroindustrie, Frankfurt a.M. 1989, S. 226 f.; H. Homburg, Rationalisierung und Industriearbeit. Arbeitsmarkt - Management - Arbeiterschaft im Siemens-Konzern Berlin 1900-1939, Berlin 1991, S. $141 \mathrm{ff}, 364 \mathrm{ff}$. 
Willms' Ansatz zur Analyse der reichsweiten Berufszählungen, dem die Theorie eines segmentierten Arbeitsmarktes zugrunde liegt. Teilarbeitsmärkte werden auf der Angebotsseite u.a. durch die Merkmale Geschlecht, Alter und Qualifikation bestimmt. Eine diesen Kriterien folgende selektive Rekrutierung von Arbeitskräften durch die Betriebe bedeutet, daß die Teilarbeitsmärkte nicht für alle Personen gleichermaßten zugänglich sind..$^{10}$ Werden die Daten der reichsweiten Betriebszählungen von 1895, 1907, 1925 und 1933 auch im Hinblick auf die Kategorie Geschlecht analysiert, dann zeigt sich, daß das Ausmaß der geschlechtsspezifischen Arbeitsmarktsegregation im Metallsektor der Verdrängungsthese jedweden Boden entzieht.

\section{Datengrundlage}

Die Analyse der Arbeitsmarktentwicklung erfolgt anhand der Betriebszählungsdaten. Die Daten geben Auskunft über den zu einem bestimmten Zeitpunkt real gegebenen Beschäftigungsstand der Betriebe. Die Erfassungsbögen wurden von den Betriebsleitungen ausgefüllt, während die Daten der Berufszählungen individuell erhoben wurden. Die Betriebszählungen sind daher vermutlich die zuverlässigere Quelle für die Analyse der geschlechtsspezifischen Beschäftigungsentwicklung in einem Industriebereich, da der potentielle Manipulationseffekt im Zuge der Datenerhebung und -auswertung geringer zu veranschlagen ist."

Eine intertemporäre Analyse der Reichsstatistiken konfrontiert mit einer Reihe von Problemen. ${ }^{12}$ Der unterschiedliche Gebietsstand der Zählungen ist ein Problem von geringerer Bedeutung. ${ }^{13}$ Die für die Beschäftigung von Frauen relevanten Wirtschaftsgruppen der Metallindustrie, Eisen-, Stahl- und Metallwarenherstellung, Maschinen-, Apparate- und Fahrzeugbau sowie Elektrotechnische Industrie einschließlich Feinmechanik und Optik, weisen einen im Vergleich zur gesamten Wirtschaftsabteilung Industrie und Handwerk unterdurchschnittlichen und relativ gleichmäßigen Verlust an Beschäftigten auf. ${ }^{14}$

Entscheidender für die Untersuchung der Arbeitsmarktentwicklung im Zeitraum 1895 bis 1933 sind die Änderungen in der Zählsystematik ab 1925.15 Bis 1907 wurde lediglich zwi-

10 Siehe Willms-Herget 1985, S. 51 ff.; außerdem: M. Kleber, Arbeitsmarktsegmentation nach dem Geschlecht. Eine kritische Analyse ökonomischer Theorien über Frauenarbeit und Frauenlöhne, München 1988 , bes. S. $144 \mathrm{ff}$.

11 Vgl. B. Hill, Women, Work and the Census: a Problem for Historians of Women, in: History Workshop Journal 35, 1993, S. 78-94. Die Autorin beschreibt am Beispiel der individuell erhobenen britischen und US-amerikanischen Zensusdaten, wie besonders Aussagen zur Entwicklung der Frauenerwerbsarbeit erschwert werden, da die Datenerhebung erheblich durch geschlechtsspezifische Stereotype und Vorannahmen der Statistiker beeinflußt und verfälscht wurde.

12 Ausführlich dazu Willms-Herget 1980, 1985; W. Maller/A. Willms/J. Handl, Strukturwandel der Frauenarbeit 1880-1980, Frankfurt a.M. 1983.

13 F. Grumbach/G. Greve, Wandlungen in der Beschäftigungsstruktur, in: $H$. Konig ( $H g$.$) , Wandlungen der$ Wirtschaftsstruktur in der Bundesrepublik Deutschland, Berlin 1962; R. Stockmann/A. Willms-Herget, Erwerbsstatistik in Deutschland. Die Berufs- und Arbeitsstättenzählungen seit 1875 als Datenbasis einer Sozialstrukturanalyse, Frankfurt a.M. 1985, S. 17.

14 Differenzen in der Anzahl der 1907 ermittelten Betriebe und Beschäftigten nach dem Gebietsstand von 1907 und 1925: Eisen-, Stahl- und Metallwarenherstellung -8,1 Prozent bzw. -6,0 Prozent; Maschinen-, Apparate- und Fahrzeugbau -6,4 Prozent bzw. -5,6 Prozent; Elektrotechnische Industrie inklusive Feinmechanik und Optik -6,4 Prozent bzw. -3,3 Prozent. Der Durchschnittswert für Industrie und Handwerk beträgt für die Beschäftigten -8,1 Prozent. Statistik des Deutschen Reiches, Bd. 413, H. 1, S. 246.

15 Siehe hierzu in erster Linie Stockmann/Willms-Herget 1985. 
schen Metallverarbeitung und Maschinenindustrie unterschieden. Ab 1925 differenzierte die Systematik in Reaktion auf die technische Entwicklung und die zunehmende Spezialisierung der Metallindustrie vier Wirtschaftsgruppen: Eisen- und Metallgewinnung, Eisen-, Metallund Stahlwarenherstellung, Maschinen-, Apparate- und Fahrzeugbau sowie Elektrotechnische Industrie einschließlich Feinmechanik und Optik. Um eine langfristige Entwicklung herausarbeiten zu können, müssen zuerst sinnvolle Vergleichskategorien gefunden werden. Zwar haben Willms und Stockmann für die Untersuchung des langfristigen Strukturwandels der Frauenarbeit von 1880 bis 1980 bereits ein neues Klassifikationsschema entwickelt. ${ }^{16}$ Es empfiehlt sich jedoch, dieses für die Untersuchung eines kürzeren Zeitraums und eines bestimmten industriellen Sektors zu modifizieren und zu verfeinern.

Willms/Stockmann haben z.B. für den Metallsektor die Eisen- und Metallgewinnung einerseits und die Eisen-, Stahl- und Metallwarenherstellung andererseits zur Analysekategorie „Eisen- und Nichtedelmetallerzeugung, Gießerei und Stahlverformung" zusammengefaßt. Diese Wirtschaftsbereiche haben jedoch für Männer und Frauen eine sehr unterschiedliche Bedeutung. Der erste Bereich steht dem Bergbau nahe und hat für die Frauenbeschäftigung kaum Relevanz. 1925 betrug der Frauenanteil in der Gewerbegruppe V (Eisen- und Metallgewinnung) der Reichsstatistik lediglich 3,5 Prozent. Der zweite Bereich umfaßt dagegen Wirtschaftszweige, die für die Frauenerwerbsarbeit im Metallsektor zentral sind, wie z.B. die Herstellung von Nägeln und Schrauben, von Knöpfen und anderen Kleinmetallwaren. In der Gewerbegruppe VI (Herstellung von Eisen-, Stahl- und Metallwaren) waren 1925 zu 16,1 Prozent Frauen beschäftigt. ${ }^{17}$ Die Zusammenfassung der beiden Wirtschaftszweige verdeckt somit die geschlechtsspezifische Arbeitsmarktsegregation.

Ein Klassifikationsschema, aus dem die geschlechtsspezifische Arbeitsmarktsegmentation über einen längeren Zeitraum möglichst genau erfaßt werden kann, verlangt eine Zusammenfassung der Wirtschaftszweige auf dem Niveau des kleinsten gemeinsamen Nenners der Zählungen von 1895 bis 1933. Es sollte u.a. die Differenzierung zwischen Konsum- und Investitionsgüterindustrien innerhalb des Metallsektors ermöglichen, um beispielsweise überprüfen zu können, ob in der Weltwirtschaftskrise Männer stärker von Arbeitslosigkeit bedroht waren, weil sie in erster Linie im Investitionsgüterbereich beschäftigt waren, und ob Frauen weniger bedroht waren, weil sie sich auf die Konsumgüterindustrien konzentrierten. ${ }^{18}$ Für meine Analyse habe ich daher eine an die Systematik der Betriebszählung von 1925 angelehnte Klassifikation zugrunde gelegt. ${ }^{19}$

16 Siehe Willms 1980, S. 39* ff.; R. Stockmann, Ein Klassifikationsschema für den Vergleich der deutschen Arbeitsstättenzählungen von 1875 bis 1970, in: Historical Social Research 32, 1984, S. 59-84; Stockmann/Willms-Herget 1985, S. $210 \mathrm{ff}$.

17 Berechnet nach Statistik des Deutschen Reiches, Bd. 413, H. 3, S. 30.

18 Zur Differenzierung von Investitions- und Konsumgüterindustrien siehe $R$. Sellien/H. Sellien (Hg.), Gablers Wirtschafts-Lexikon, Wiesbaden ${ }^{6} 1965$; außerdem: W. Woytinsky, Der deutsche Arbeitsmarkt. Ergebnisse der gewerkschaftlichen Arbeitslosenstatistik 1919-1929, Bd. 1, Berlin 1930, S. 33.

19 Das zugrunde gelegte Klassifikationsschema ist in meiner Dissertation ausführlich dokumentiert. Originaldaten in: Statistik des Deutschen Reiches, Bd. 113, Tab. 2, S. 112 ff. u. Tab. 3, S. 132 ff.; Bd. 213, Abt. I, H. 1, Tab. 2, S. 44 ff.; H. 2, Tab. 3, S. 70 ff.; Bd. 413, T. I, Tab. 2, S. 274 ff. u. T. II, Tab. 3, S. 12 ff.; Bd. 462, H. 2, Tab. 1, S. 15 ff. u. Tab. 3, S. 58 ff. 


\section{Beschäftigungsentwicklung im Metallsektor}

Der durch die vier reichsweiten Betriebszählungen erfaßte Untersuchungszeitraum läßt sich im Hinblick auf die Arbeitsmarktentwicklung in drei Phasen gliedern. In der Phase der Hochindustrialisierung bis zum Ersten Weltkrieg ist eine beträchtliche Ausweitung der weiblichen Beschäftigung im Metallsektor festzustellen, die sich aus dem Zusammenspiel erhöhter Nachfrage nach Arbeitskräften und der Suche nach lukrativen Beschäftigungsmöglichkeiten seitens weiblicher Arbeitskräfte ergab. Im Ersten Weltkrieg kam es infolge des Arbeitskräftebedarfs der Kriegswirtschaft zu einer Umschichtung auf dem weiblichen Arbeitsmarkt, die zu einer beschleunigten Zunahme weiblicher Arbeitskräfte in der Metallindustrie führte. Die Arbeitsmarktentwicklung der Weimarer Republik war zunächst durch das Bemühen um Wiederherstellung der geschlechtsspezifischen Arbeitsmarktverhältnisse der Vorkriegszeit geprägt. Seit der Inflation 1923/24 entwickelte sich die Männer- und Frauenbeschäftigung in der Metallindustrie weitgehend parallel. ${ }^{20}$

Tabelle 1: Anteil der im Metallsektor Beschäftigten an der Wirtschaftsabteilung Industrie und Handwerk

(Ind. \& Handwerk $=100$ )

\begin{tabular}{|r|r|r|r|}
\hline & Beschäftigte & Frauen & Männer \\
\hline 1895 in Prozent & 16,8 & 3,9 & 20,0 \\
abs. & 1.334 .046 & 60.234 & 1.273 .812 \\
1907 in Prozent & 20,4 & 6,3 & 24,0 \\
abs. & 2.195 .002 & 136.753 & 2.058 .249 \\
1925 in Prozent & 26,3 & 13,8 & 30,0 \\
abs. & 3.338 .072 & 399.798 & 2.938 .274 \\
1933 in Prozent & 21,0 & 11,0 & 24,3 \\
abs. & 1.843 .730 & 241.548 & 1.602 .182 \\
\hline
\end{tabular}

Die Industrialisierung des 19. Jahrhunderts wirkte sich auf Männer- und Frauenbeschäftigung unterschiedlich aus. Die Metallindustrie als Leitsektor der Industrialisierung gewann für die Frauenlohnarbeit erst mit zeitlicher Verzögerung größere Relevanz. Zu den bevorzugten Bereichen weiblicher Erwerbsarbeit im 19. Jahrhundert gehörten zweifellos die Textilindustrie und das Bekleidungsgewerbe. ${ }^{21}$ Die verspätete Einbeziehung von Frauen in den Metallsektor ist an der Betriebszählung von 1895 abzulesen (Tab. 1). Nur 3,9 Prozent (60.234) aller in Industrie und Handwerk beschäftigten Frauen waren in der Metallindustrie tätig. Demgegenüber arbeitete ein Fünftel aller Industriearbeiter und Handwerker im Metallsektor (1.273.812). Die Bedeutung dieses industriellen Sektors für die Frauenarbeit nahm jedoch kontinuierlich zu, so daß 1925 schließlich 13,8 Prozent aller weiblichen Beschäftigten in Industrie und Handwerk in der Metallindustrie arbeiteten. Die Zahlen für die männlichen

$20 \mathrm{Um}$ diese Phase im einzelnen nachzuzeichnen, muß auf die Daten der Gewerbeaufsicht zurückgegriffen werden. Siehe dazu B. Kassel, Das ,schwache Geschlecht' und seine Kleider. Technisierung und weibliche Fähigkeiten und Fertigkeiten in der Metallindustrie (1900-1930), in: G. Hurrle/F. J. Jelich/J. Seitz (Hg.), Wie bedingen sich Arbeit, Technik und Beruf im industriellen Prozeß?, Marburg 1994, S. 80-92.

$21 \mathrm{Zu}$ früheren Beispielen von Frauenarbeit im Metallbereich siehe z.B. R. Bake, Die Arbeits- und Lebensweise von Manufakturarbeiterinnen im 18. Jahrhundert, in: H. Pohl/W. Treue (Hg.), Die Frau in der deutschen Wirtschaft, Wiesbaden 1985, S. 54-64. 
Beschäftigten belegen nicht, daß die weibliche Beschäftigungsentwicklung zu Lasten der Männer ging. 1925 erreichte der Anteil der männlichen Metallbeschäftigten fast ein Drittel aller männlichen Beschäftigten in Industrie und Handwerk.

Die relativ stärkere Zunahme der Frauenbeschäftigung in der Metallindustrie (1895: 3,9 Prozent; 1925: 13,8 Prozent) dürfte vor allem auf den verspäteten Eintritt in diesen industriellen Sektor zurückzuführen sein. Um der Entwicklung im einzelnen auf die Spur zu kommen, müssen diese Zahlen nach Wirtschaftszweigen und Berufsstellungen genauer aufgeschlüsselt werden.

Tabelle 2: Frauenanteil in den einzelnen Wirtschaftsgruppen der Metallindustrie (in Prozent und abs. Zahl der Frauen)

\begin{tabular}{|r|c|c|c|c|c|}
\hline & Metallindustrie & $\begin{array}{c}\text { Metall- } \\
\text { gewinnung }\end{array}$ & $\begin{array}{c}\text { Metallwaren- } \\
\text { industrie }\end{array}$ & $\begin{array}{c}\text { Maschinen- } \\
\text { bauindustrie }\end{array}$ & $\begin{array}{c}\text { Elektrotechn. } \\
\text { Industrie }\end{array}$ \\
\hline 1895 in Prozent & 4,5 & 2,8 & 7,5 & 1,0 & 7,6 \\
abs. & 60.234 & 6.914 & 42.626 & 4.321 & 6.373 \\
1907 in Prozent & 6,2 & 2,7 & 10,8 & 1,5 & 14,3 \\
abs. & 136.753 & 10.658 & 85.190 & 11.731 & 29.174 \\
1925 in Prozent & 12,0 & 3,8 & 17,3 & 5,8 & 24,8 \\
abs. & 399.798 & 22.852 & 156.483 & 72.254 & 148.209 \\
1933 in Prozent & 13,1 & 3,7 & 16,3 & 7,6 & 24,6 \\
abs. & 241.548 & 10.031 & 98.110 & 46.559 & 86.848 \\
\hline
\end{tabular}

Tabelle 2 bestätigt den aus Tabelle 1 abzulesenden Trend, daß sich weibliche Arbeitskräfte im Metallsektor allmählich etablierten. Die Zahlen deuten außerdem darauf hin, daß der Erste Weltkrieg die Zunahme weiblicher Beschäftigung beschleunigte. Jedoch hatten die einzelnen Wirtschaftsgruppen an dieser Entwicklung ganz unterschiedlich teil. Besonders ausgeprägt sind die Steigerungen des Frauenanteils in der Eisen-, Stahl- und Metallwarenverarbeitung (1907 zu 1925 von 10,8 Prozent auf 17,3 Prozent) sowie in der Elektrotechnischen Industrie einschließlich Feinmechanik und Optik (1907 zu 1925 von 14,3 Prozent auf 24,8 Prozent). Auch ein Frauenanteil im Maschinenbau von 5,8 Prozent im Jahre 1925 ist angesichts der um die Jahrhundertwende marginalen Rolle von Frauen in diesem Sektor bemerkenswert.

Die herausragende Bedeutung der Elektrotechnischen Industrie für die Nachfrage nach weiblichen Arbeitskräften zeigt sich in den absoluten Zahlen. Gegen Ende des 19. Jahrhunderts beschäftigte diese noch sehr junge Industrie erst wenig Arbeitskräfte. Die mit der Verbreitung des elektrischen Antriebs einsetzende erhebliche Expansion erhöhte die Nachfrage nach Arbeitskräften. Frauen profitierten davon offensichtlich stärker als Männer. Schaut man sich außerdem die geschlechtsspezifischen Steigerungsquoten der Beschäftigung differenziert nach Wirtschaftsgruppen an (Tab. 3), ist indes zweifelsfrei festzustellen, daß diese Entwicklung kaum zu Lasten von Männern gegangen sein kann.

Die Steigerung der weiblichen Beschäftigung in der Elektrotechnischen Industrie zwischen 1895 und 1907 um mehr als 350 Prozent ist in der Tat beeindruckend und läßt die Zunahme männlicher Beschäftigung um ca. 125 Prozent in diesem Sektor eher bescheiden erscheinen. Doch es darf nicht außer acht gelassen werden, daß es sich hier um einen Basiseffekt bei niedrigen Ausgangszahlen für weibliche Arbeitskräfte handelt: 1895 beschäftigte die Elek- 
trotechnische Industrie lediglich 6.373 Frauen im Vergleich zu 78.019 Männern, 1907 waren es 29.174 Frauen und 175.367 Männer.

Tabelle 3: Beschäftigungsentwicklung 1895-1933

(in Prozent)

\begin{tabular}{|r|c|c|c|}
\hline & $1895-1907$ & $1907-1925$ & $1925-1933$ \\
\hline Industrie \& Handwerk & $+35,5$ & $+18,2$ & $-30,8$ \\
Männer & $+34,7$ & $+14,3$ & $-32,7$ \\
Frauen & $+39,1$ & $+33,9$ & $-24,5$ \\
\hline Metallindustrie & $+64,5$ & $+52,1$ & $-44,8$ \\
Männer & $+61,6$ & $+42,8$ & $-45,5$ \\
Frauen & $+127,0$ & $+192,4$ & $-39,6$ \\
\hline Männer & $+65,3$ & $+47,9$ & $-54,0$ \\
Frauen & $+65,7$ & $+46,1$ & $-54,0$ \\
Männer & $+54,2$ & $+114,4$ & $-56,1$ \\
\hline Frauen & $+38,3$ & $+14,3$ & $-33,1$ \\
Männer & $+33,3$ & $+6,0$ & $-32,2$ \\
Frauen & $+99,9$ & $+83,7$ & $-37,3$ \\
\hline Metallwarenindustrie & $+83,5$ & $+55,5$ & $-50,6$ \\
Männer & $+82,6$ & $+48,6$ & $-51,5$ \\
Frauen & $+171,5$ & $+515,9$ & $-35,6$ \\
\hline Maschinenbauindustrie & $+142,4$ & $+192,8$ & $-41,4$ \\
Elektrotechnische Ind. & $+124,8$ & $+157,0$ & $-41,0$ \\
\hline
\end{tabular}

In Tabelle 3 ragen darüber hinaus die Steigerungsquoten der Frauenarbeit im Bereich des Maschinenbaus mit gut 170 Prozent (1895-1907) und mehr als 500 Prozent (1907-1925) heraus. Auch hier handelt es sich jedoch um Basiseffekte. 1895 beschäftigte diese Wirtschaftsgruppe lediglich 4.321 Frauen neben 430.651 Männern, und auch 1925 blieben die 72.254 Frauen gegenüber den 1.168.247 Männern im Maschinenbau eine verschwindend kleine Gruppe.

Die Wirtschaftsgruppe Maschinenbau umfaßte z.B. den Bau von Dampfmaschinen, Lokomotiven, Zentralheizungsanlagen und Großmaschinen u.a. für die Textilindustrie, Druckindustrie und Wäschereien. Solche Maschinen zeichneten sich durch besondere Größe, Gewicht und komplizierte Mechanik aus. In welchem Maße diese Attribute männlich konnotiert waren, schlug sich nicht zuletzt in der Werbung für Produkte der Metallindustrie in den technischen Fachzeitschriften der zwanziger Jahre nieder. Die „geschlechtsspezifische Arbeitsteilung " war hier augenfällig: Frauen warben für kleine Schrauben und Muttern aus der Massenfabrikation, Männer für riesige, in Einzelfertigung hergestellte Schiffsschrauben. Diese Werbestrategien spiegeln die geschlechtsspezifische Arbeitsteilung in der Herstellung der verschiedenen Produkte der Maschinenbauindustrie. 1925 waren 15,6 Prozent der knapp 30.000 in der Herstellung von Nähmaschinen und Nähmaschinenteilen Beschäftigten weiblich, der Frauenanteil an den gut 20.000 in der Herstellung von Rechen- und Schreibmaschinen Beschäftigten betrug 20,4 Prozent, in der Produktion landwirtschaftlicher Maschinen waren indes unter den fast 55.000 Beschäftigten nur 3,5 Prozent Frauen.

Da die Zahlen der Reichsstatistik in keiner Publikation des DMV auf eine ähnlich differenzierte Weise betrachtet wurden, die Wahrnehmung von Frauenarbeit vielmehr immer 
durch die hohen allgemeinen Zahlen bestimmt war, mögen Steigerungsquoten wie die obengenannten durchaus bedrohlich gewirkt haben. Dies gilt um so mehr für hochgradig männlich definierte Wirtschaftszweige. Der Einbruch von Frauen in diese Männerwelt konnte leicht als Bedrohung für ein männliches Selbstbild, das sich auf Körperkraft und physische Wirksamkeit gründete, interpretiert werden. ${ }^{2}$

Gilt dies schon für konjunkturell günstige Zeiten, so muß die Beschäftigungsentwicklung in den Krisenjahren der Weimarer Republik erst recht alarmierend gewirkt haben. Die Daten der Reichsstatistik spiegeln weder das Auf und Ab der Beschäftigungsentwicklung im Zuge von Krieg, Demobilmachung und Inflation, noch vermögen sie die Entwicklung unter dem Einfluß von Stabilisierungskrise, Rationalisierung und Weltwirtschaftskrise adäquat wiederzugeben. Tabelle 3 vermittelt den Eindruck, Frauen in Industrie und Handwerk seien in geringerem Maße vom Rückgang der Beschäftigung 1925 bis 1933 betroffen gewesen. Insgesamt sah die Beschäftigungsentwicklung für die männlichen und weiblichen Metallbeschäftigten schlechter aus als für den Durchschnitt der Industriebeschäftigten. Innerhalb des Metallsektors waren die Beschäftigungsverluste für Frauen offensichtlich geringer als für Männer. ${ }^{23}$ Gleichwohl ist die Erwerbslosigkeit von Frauen nicht zu unterschätzen. Die prozentuale Abnahme der männlichen und weiblichen Beschäftigten lag zwischen 1925 und 1933 viel enger beieinander lagen als die Zuwachsraten der Jahre zuvor. Möglicherweise näherten sich im längeren Verlauf der Krise männliche und weibliche Erwerbschancen einander an. Die Produktionsgüterindustrien als Schwerpunkt der Männerarbeit litten früher und stärker als die Konsumgüterindustrien unter der Weltwirtschaftskrise. Im Konsumbereich führte der Produktionsrückgang oft erst über Kurzarbeit in die Erwerbslosigkeit. ${ }^{24}$

\section{Situssegregation}

Die Tabellen 1-3 haben die unterschiedliche Relevanz der einzelnen Wirtschaftsgruppen für Frauenarbeit in der Metallindustrie gezeigt. Noch deutlicher läßt sich die geschlechtsspezifische Verteilung der Arbeitskräfte graphisch darstellen.

Zunächst fällt auf, daß die männlichen Beschäftigten sich 1895 und 1907 sehr viel gleichmäßiger auf die Wirtschaftsgruppen der Metallindustrie verteilten als die weiblichen. 1895 arbeitete noch nicht einmal die Hälfte aller männlichen Metallbeschäftigten in der Herstellung von Eisen-, Stahl- und Metallwaren, für die Frauen betrug der Anteil dagegen 70 Prozent. Der Maschinenbau war für Männer mit einem Anteil von einem Drittel der zweitwichtigste Beschäftigungsbereich. Allein die Zahl der Männer in diesem Sektor übertraf mit

$22 \mathrm{Vgl}$. C. Cockburn, Machinery of Dominance. Women, Men and Technical Know-How, London 1985, S. 194; H. Kern/M. Schumann, Industriearbeit und Arbeiterbewußtsein. Eine empirische Untersuchung über den Einfluß der aktuellen technischen Entwicklung auf die industrielle Arbeit und das Arbeiterbewußtsein, Frankfurt a.M. ${ }^{2} 1985$, S. 23 f.; siehe auch z.B. M. Mende, „Männer des Feuers und der eisernen Kraft“. Die Arbeit des Schmieds, in: W. Ruppert (Hg.), Arbeiter. Lebensformen, Alltag und Kultur, München 1986, S. $224-235$.

23 Ein günstigeres Bild für die weibliche Beschäftigungsentwicklung in der Metallindustrie vermittelt auch die Berufszählung von 1933. Allerdings ist hier ein hoher Anteil von nicht gemeldeten weiblichen Erwerbslosen zu berücksichtigen. Siehe zur Problematik der Arbeitslosenstatistik $S$. Rouette, Die Realitäten der Zahlen. Arbeitsmarktstatistik und Politik zu Beginn der Weimarer Republik, in: Jahrbuch für Wirtschaftsgeschichte 1993, S. 133-153.

24 Homburg 1991, S. 374 ff. exemplarisch zur unterschiedlichen Entwicklung von Investitions- und Konsumgüterbereich in der Krise anhand der Beschäftigtenzahlen bei SSW und S\&H 
430.651 die Zahl aller weiblichen Beschäftigten in der Metallindustrie (60.234) um ein Vielfaches.

Abbildung 1: Von 100 beschäftigten Männern bzw. Frauen der Metallindustrie arbeiteten in den einzelnen Metallwirtschaftsgruppen ${ }^{s}$

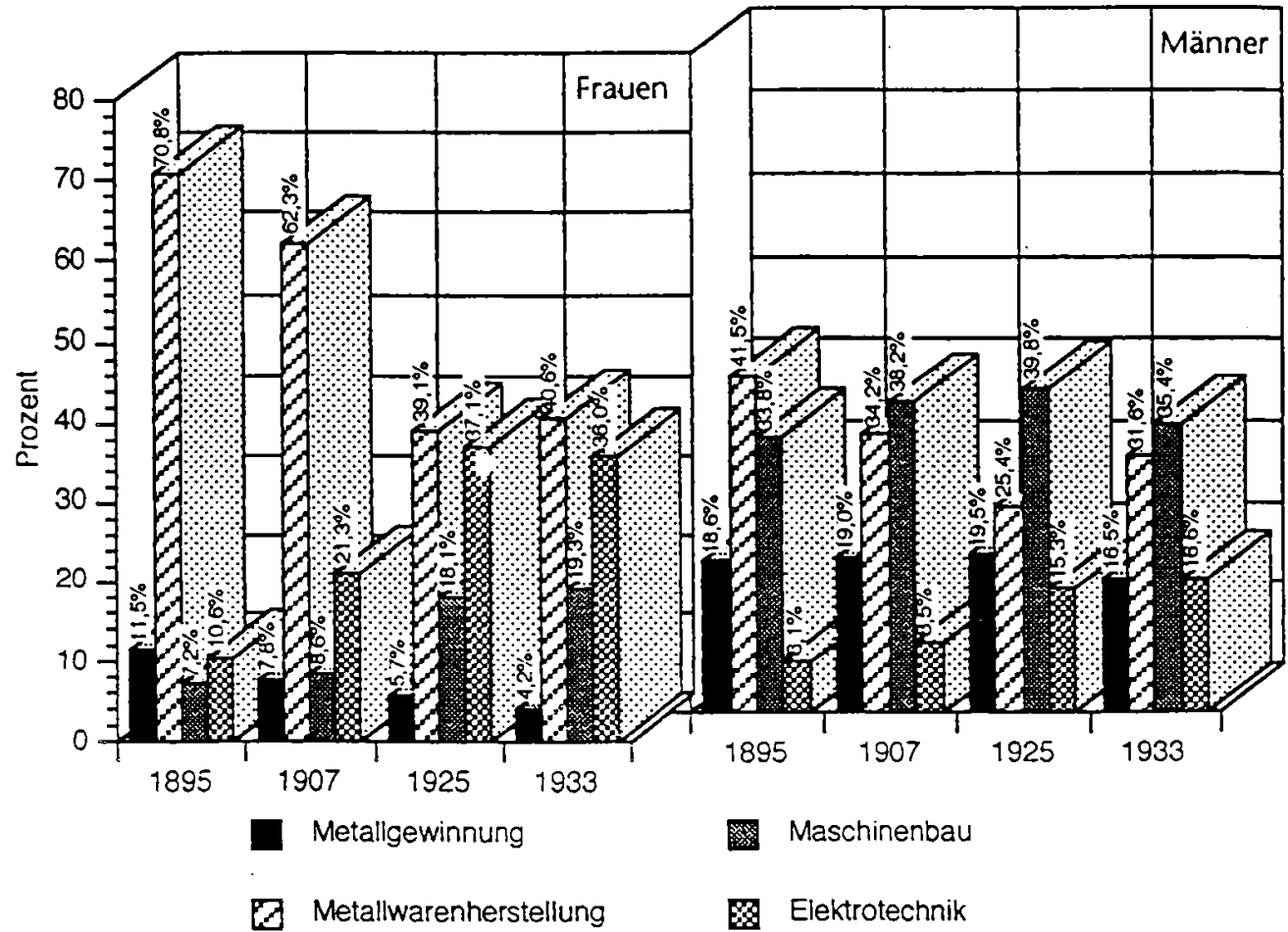

- Quelle: Eigene Berechnungen auf der Basis der Betriebszählungen 1895, 1907, 1925 und 1933.

1907 war die Herstellung von Eisen-, Stahl- und Metallwaren noch immer der mit Abstand wichtigste Beschäftigungsbereich für Frauen, doch immerhin schon ein gutes Fünftel der weiblichen Beschäftigten entfiel jetzt auf die Elektrotechnische Industrie. Darin spiegelt sich der erhöhte Arbeitskräftebedarf einer modernen Industrie (siehe auch Tab. 3). 1925 beschäftigte schließlich selbst der Maschinenbau fast ein Fünftel aller Frauen in der Metallindustrie. Daß damit jedoch die männliche Vorherrschaft nicht in Gefahr war, zeigt der Frauenanteil innerhalb der Wirtschaftsgruppe, der auch in den zwanziger Jahren weit unter 10 Prozent lag. Dagegen unterstreicht ein Frauenanteil von etwa 25 Prozent in der Elektrotechnischen Industrie deren überragende Bedeutung für den weiblichen Metallarbeitsmarkt (Tab. 2). Die Entwicklung ging insgesamt dahin, daß die Verteilung der weiblichen Beschäftigten auf die Wirtschaftsgruppen der Metallindustrie 1925 und 1933 wesentlich ausgeglichener war als in der Vorkriegszeit. 
Die Veränderungen auf dem Metallarbeitsmarkt für Männer springen nicht so sehr ins Auge. Allerdings ist auch hier der relative Bedeutungsverlust der Metallwarenindustrie zugunsten der Elektrotechnischen Industrie zu erkennen. Von größter Bedeutung ist indes der Maschinenbau, in dem 1925 nahezu zwei Fünftel der männlichen Metallbeschäftigten (1.168.247) arbeiteten. In diesem ökonomisch und arbeitsmarktpolitisch wichtigsten Bereich der Metallindustrie blieben Männer überwiegend unter sich: Der Frauenanteil lag bei 5,8 Prozent (72.254). ${ }^{25}$ Dies machte die männliche Arbeitsmarktposition andererseits relativ verletzlicher, wie die Grafik mit den Werten für 1933 veranschaulicht (auch Tab. 3). Der Maschinenbau als überwiegend zum Produktionsgüterbereich zählende Industrie litt stärker als die für die Frauenbeschäftigung wichtigen Wirtschaftsgruppen unter dem Beschäftigungsrückgang während der Weltwirtschaftskrise. ${ }^{26}$ Mit dem Rückgang der Zahl männlicher Arbeitskräfte im Maschinenbau von 1.168.247 im Jahre 1925 auf 566.652 im Jahre 1933 gewann vor allem die Metallwarenindustrie wieder an relativer Bedeutung auf dem männlichen Metallarbeitsmarkt, da dort die Beschäftigung weniger stark zurückging.

Blickt man nun noch einmal auf die weibliche Beschäftigungsentwicklung, so könnte zunächst erstaunen, daß die Depression sich offenbar nicht in ähnlicher Weise auf die Verteilung der Frauen auf die Metallwirtschaftsgruppen auswirkte - im Gegenteil, für den weiblichen Arbeitsmarkt scheint der Maschinenbau 1933 im Vergleich zu 1925 leicht an Bedeutung gewonnen zu haben (Abb. 1). Tabelle 3 belegt außerdem, daß die weiblichen Beschäftigten des Maschinenbaus zwischen 1925 und 1933 mit -35,6 Prozent den geringsten Beschäftigungsrückgang in der Metallindustrie insgesamt erlebten. Der Durchschnittswert auf dem weiblichen Metallarbeitsmarkt betrug -39,6 Prozent.

Dieser Befund scheint der These zu widersprechen, daß die Beschäftigten um so stärker von Erwerbslosigkeit bedroht waren, je näher ihre Wirtschaftsgruppe gemessen am Produktionsspektrum zum Investitionsgüterbereich stand. Tabelle 3 weist jedoch einige Indizien für die grundsätzliche Richtigkeit der These auf. Der Beschäftigungsrückgang von 1925 bis 1933 war in der Metallindustrie, die in ihrer Gesamtheit vorrangig dem Investitionsgüterbereich zuzuordnen ist, sehr viel größer als im industriellen Durchschnitt. Sowohl in Industrie und Handwerk als auch im gesamten Metallsektor erfaßte der Beschäftigungsrückgang Frauen in relativ geringerer Zahl als Männer. Allerdings lagen die Beschäftigungsverluste mit 45,5 Prozent bei den Männern und 39,6 Prozent bei den Frauen recht nahe beieinander. Der weibliche Beschäftigungsrückgang in der gesamten Metallindustrie fiel deshalb etwas geringer aus, weil Frauen in den Investitionsgüterbereichen Metallgewinnung und Maschinenbau, d.h. in den Wirtschaftsgruppen mit den größten Beschäftigungsverlusten von 54 bzw. 50,6 Prozent, absolut gesehen nur marginal vertreten waren (Tab. 2).

Das positivere Bild, das die geschlechtsspezifische Beschäftigungsentwicklung für die Metallindustrie insgesamt vom weiblichen Arbeitsmarkt vermittelt, kehrte sich allerdings in der Metallgewinnung ebenso um wie in der Metallwaren- und der Elektrotechnischen Indu-

251925 beschäftigte die Maschinenbauindustrie ca. 1,3 Mio Arbeitskräfte. In der Metallwarenindustrie lag der Beschäftigungsstand bei etwa 900.000. Nur etwa 500.000 Arbeitskräfte waren in der Metallgewinnung und ca. 600.000 in der Elektrotechnik beschäftigt. StDR 413, H. 3, S. 30.

26 H.A. Winkler, Der Weg in die Katastrophe. Arbeiter und Arbeiterbewegung in der Weimarer Republik 1930 bis 1933, Berlin 1987, S. 59 ff. zu Auswirkungen der Depression auf Investitionsgüterindustrien im Vergleich zu Verbrauchsgüterindustrien; W. Fischer, Bergbau, Industrie und Handwerk 1914-1970, in: H. Aubin/W. Zorn $(\mathrm{Hg}$.), Handbuch der Deutschen Wirtschafts- und Sozialgeschichte, Bd. 2, Stuttgart 1976, S. 812. 
strie. In allen drei Bereichen waren Frauen stärker als Männer vom Beschäftigungsabbau betroffen (Tab. 3). Das bedeutet, daß allein die vergleichsweise günstige weibliche Beschäftigungsentwicklung im Maschinenbau für die geringeren Beschäftigungsverluste für Frauen in der Gesamtindustrie verantwortlich war. Der Beschäftigungsabbau im Maschinenbau betrug für Männer 51,5 Prozent, für Frauen aber lediglich 35,6 Prozent.

\section{Arbeitskräftestruktur}

Sobald die Analyse auf nach Berufsstellungen abgegrenzte Teilarbeitsmärkte zielt, kann dieser beträchtliche Unterschied erklärt werden. Die Maschinenbauindustrie war in hohem Maße großbetrieblich organisiert. Die mit der Entwicklung zum Großbetrieb schnell ausgedehnten Verwaltungsapparate beschäftigten vor allem in den kaufmännischen Büros außerordentlich viele weibliche Angestellte. ${ }^{27}$ Die Zahl von nur ca. 1.000 weiblichen Angestellten in der Metallindustrie im Jahre 1895 verzehnfachte sich bis 1907. Der Frauenanteil unter den Angestellten in der Metallindustrie stieg im gleichen Zeitraum von 3 Prozent auf 12 Prozent. Betrachtet man die Beschäftigungsentwicklung in der Metallindustrie differenziert nach Berufsstellungen, wird sehr deutlich, daß die Zunahme der weiblichen Arbeitskräfte zwischen 1907 und 1925 zu einem großen Teil auf die Ausweitung der Angestelltentätigkeit zurückzuführen ist. Wie Tabelle 4 darüber hinaus zeigt, waren Angestellte während der Wirtschaftskrise in viel geringerem Umfang vom Stellenabbau betroffen als Arbeiterinnen und Arbeiter. ${ }^{23}$

Dies schlägt in der Maschinenbau- und in der gleichfalls großbetrieblich geprägten Elektrotechnischen Industrie nicht gleichermaßen zu Buche, da in der Elektrotechnik sehr viel mehr Frauen im Produktionsbereich arbeiteten. Tabelle 5 belegt dies für die Jahre 1925 und 1933. In den ausgeprägtesten Männerdomänen der Metallindustrie, also in der Metallgewinnung und im Maschinenbau, war ein wesentlich größerer Anteil der weiblichen Beschäftigten als Angestellte tätig, als dies in Metallwaren- und Elektrotechnischer Industrie der Fall war.

27 Die marginale Existenz weiblicher Angesteliter in der Industrie bis zum Ersten Weltkrieg findet in der Forschung ihre Entsprechung. Zu Angestellten: T. Pierenkemper, Arbeitsmarkt und Angestellte im deutschen Kaiserreich 1880-1913. Interessen und Strategien als Elemente der Integration eines segmentierten Arbeitsmarktes, Stuttgart 1987, bes. S. 41 f., 194 ff.; ders., Allokationsbedingungen im Arbeitsmarkt. Das Beispiel des Arbeitsmarktes für Angestelltenberufe im Kaiserreich, 1880-1913, Opladen 1983; H.J. Rupieper, Arbeiter und Angestellte im Zeitalter der Industrialisierung. Eine sozialgeschichtliche Studie am Beispiel der Maschinenfabriken Augsburg und Nürnberg (MAN) 1837-1914, Frankfurt a.M. 1982; G. Schulz, Die Arbeiter und Angestellten bei Felten \& Guilleaume. Sozialgeschichtliche Untersuchung eines Kölner Industrieunternehmens im 19. und beginnenden 20. Jahrhundert, Wiesbaden 1979; J. Kocka, Unternehmensverwaltung und Angestelltenschaft am Beispiel Siemens 1847-1914. Zum Verhältnis von Kapitalismus und Bürokratie in der deutschen Industrialisierung, Stuttgart 1969. Zu Frauen in Angestelltenberufen: $U$. Frevert, Traditionale Weiblichkeit und moderne Interessenorganisation: Frauen im Angestelltenberuf 1918-1933, in: Geschichte und Gesellschaft 7, 1981, S. 507-533; U. Nienhaus, Berufsstand weiblich. Die ersten weiblichen Angesteltten, Berlin 1982.

$28 \mathrm{Vgl}$. H. Kramer, Frankfurt'sWorking Women: Scapegoats or Winners of the Great Depression?, in: R.J. Evans/D. Geary ( $\mathrm{Hg}$.), The German Unemployed. Experiences and Consequences of Mass Unemployment from the Weimar Republic to the Third Reich, London 1987, S. 108-141. 
Tabelle 4: Beschäftigungsentwicklung nach Berufsstellungen (in Prozent) ${ }^{\mathbf{a}}$

\begin{tabular}{|l|c|c|c|c|}
\hline \multirow{2}{*}{} & \multicolumn{2}{|c|}{$1907-1925$} & \multicolumn{2}{c|}{$1925-1933$} \\
\cline { 2 - 5 } & Frauen & Männer & Frauen & Männer \\
\hline Beschäftigte & $+192,4$ & $+42,8$ & $-40,6$ & $-45,6$ \\
Arbeiter/innen & $+164,8$ & $+40,0$ & $-46,7$ & $-52,0$ \\
Angestellte & $+589,8$ & $+118,5$ & $-34,4$ & $-40,3$ \\
\hline
\end{tabular}

- Die originale Reichsstatistik des Jahres 1933 ist nach Berufsstellungen und Wirtschaftsgruppen nicht ausreichend differenziert, so daß sich die Verteilung der Beschäftigten nach Berufsstellungen nicht nach meinem Klassifikationsschema umgruppieren läßt und sich geringfügige Differenzen zu den anderen Tabellen ergeben.

Geschlechtsspezifische Unterschiede in der Arbeitskräftestruktur zeigt Tabelle 5 außerdem bei den mithelfenden Familienangehörigen und alleintätigen Selbständigen. Männer und Frauen hatten in beiden Arbeitskräftegruppen ganz unterschiedliches Gewicht. Unter den 62.971 alleintätigen Selbständigen des Jahres 1925 gab es nur 3.821 Frauen; das entsprach einem Frauenanteil von 6,1 Prozent. Von den Mithelfenden Familienangehörigen waren im gleichen Jahr 12.935 Frauen, das heißt 40 Prozent.

Die beiden Arbeitskräftegruppen hatten darüber hinaus relativ zu den Arbeitern und Arbeiterinnen bzw. zu den Angestellten ein geschlechtsspezifisch unterschiedliches Gewicht für Männer- und Frauenarbeit. Dies scheint für die Beschäftigungsentwicklung in der Weltwirtschaftskrise wichtig. Die Zahl der alleintätigen männlichen Selbständigen nahm von 1925 (59.150) bis 1933 (109.127) um ca. 84 Prozent zu. Diese Zunahme ist vor allem der Metallwarenindustrie geschuldet, wo sich die absolute Zahl der alleintätigen männlichen Selbständigen verdoppelte (66.724). Das veränderte relative Gewicht dieser Arbeitskräftegruppe zu den Beschäftigten insgesamt in der Metallwarenindustrie - 1925 arbeiteten innerhalb der Wirtschaftsgruppe 4,5 Prozent der Männer allein selbständig, 1933 waren es indes 13,5 Prozent - läßt vermuten, daß Männer aufgrund ihrer besseren Ausbildungsvoraussetzungen wahrscheinlich eher die Möglichkeit hatten, sich als Handwerker selbständig über Wasser zu halten. In diese Interpretation paßt der Rückgang des Arbeiteranteils von 71,8 Prozent (1925) auf 57,3 Prozent (1933) in der gleichen Wirtschaftsgruppe. Daß das Überleben als Handwerker eher in Branchen möglich war, die mit einer bescheideneren technischen und maschinellen Ausstattung auskamen, liegt auf der Hand. ${ }^{29}$ Aus diesem Zusammenhang resultiert dann auch die günstigere männliche Beschäftigungsentwicklung von 1925 bis 1933 in der Metallwarenindustrie, wie sie in Tabelle 3 ausgewiesen ist: Die Zahl der beschäftigten Frauen ging um 37,3 Prozent zurück, die der beschäftigten Männer um 32,2 Prozent.

29 Ein Beispiel: Zu typischen Handwerksbetrieben in der Metallbranche dürften die Klempnereien zählen. Hier waren 1925 8,2 Prozent (8.439) der männlichen Beschäftigten alleintätige Selbständige. Von allen alleintätigen selbständigen Männern in der Metallindustrie (59.150) entfielen 14,3 Prozent allein auf Klempnereien, aber nur 15,6 Prozent (9.243) auf den gesamten beschäftigungsstarken Bereich des Maschinen-, Apparate- und Fahrzeugbaus. Wie stark die Zahl der alleintätigen Selbständigen speziell in Klempnereien bis 1933 anstieg, läßt sich aus der Zählung von 1933 mangels ausreichend differenzierter Aufbereitung der Reichsstatistik nicht herausdestillieren. Zur Bedeutung der Kleinbetriebe in der Krise siehe auch Winkler 1987, S. 97 f. 
Tabelle 5: Arbeitskräftestruktur in den Metallwirtschaftsgruppen für die Jahre 1925 und 1933 (abs.; in Prozent, Beschäftigte $=100$ )

\begin{tabular}{|c|c|c|c|c|c|c|c|c|c|c|c|c|c|c|c|c|}
\hline & \multicolumn{4}{|c|}{ Arbeiterinnen } & \multicolumn{4}{|c|}{ Angestellte } & \multicolumn{4}{|c|}{$\begin{array}{l}\text { Mithelfende } \\
\text { Familienangehörige }\end{array}$} & \multicolumn{4}{|c|}{ Alleintätige Selbstăndige } \\
\hline & \multicolumn{2}{|c|}{ Frauen } & \multicolumn{2}{|c|}{ Männer } & \multicolumn{2}{|c|}{ Frauen } & \multicolumn{2}{|c|}{ Männer } & \multicolumn{2}{|c|}{ Frauen } & \multicolumn{2}{|c|}{ Männer } & \multicolumn{2}{|c|}{ Frauen } & \multicolumn{2}{|c|}{ Männer } \\
\hline & abs. & $\begin{array}{l}\text { Pro- } \\
\text { zent }\end{array}$ & abs. & $\begin{array}{l}\text { Pro- } \\
\text { zent }\end{array}$ & abs. & $\begin{array}{l}\text { Pro- } \\
\text { zent }\end{array}$ & abs. & $\begin{array}{l}\text { Pro- } \\
\text { zent }\end{array}$ & abs. & $\begin{array}{l}\text { Pro- } \\
\text { zent } \\
\end{array}$ & abs. & \begin{tabular}{|l|} 
Pro- \\
zent \\
\end{tabular} & abs. & $\begin{array}{l}\text { Pro- } \\
\text { zent } \\
\end{array}$ & abs. & \begin{tabular}{|l|} 
Pro- \\
zent \\
\end{tabular} \\
\hline \multicolumn{17}{|l|}{1925} \\
\hline Metallwarenherstellung & 125.983 & 80,5 & 535.599 & 71,8 & 17.622 & 11,3 & 31.767 & 4,3 & 7.048 & 4,5 & 14.638 & 2,0 & 2.670 & 1,7 & 33.298 & 4,5 \\
\hline Maschinenbau & 41.040 & 56,8 & 967.296 & 82,8 & 26.650 & 36,9 & 68.180 & 5,8 & 1.900 & 2,6 & 2.486 & 0,2 & 105 & 0,2 & 9.243 & 0,8 \\
\hline Elektrotechnik & 116.741 & 78,8 & 326.548 & 72,5 & 24.043 & 16,2 & 37.144 & 8,2 & 3.957 & 2,7 & 2.227 & 0,5 & 1.041 & 0,7 & 16.590 & 3,7 \\
\hline Metallindustrie & 300.272 & 75,1 & 2.335 .512 & 79,5 & 74.241 & 18,6 & 167.313 & 5,7 & 12.935 & 3,2 & 19.433 & 0,7 & 3.821 & 1,0 & 59.150 & 2.0 \\
\hline \multicolumn{17}{|l|}{1933} \\
\hline Metallwarenherstellung & 66.292 & 71,0 & 434.313 & 57,3 & 12.889 & 13,8 & 19.653 & 4,0 & 9.105 & 9,8 & 20.531 & 4,2 & 1.441 & 1,5 & 66.724 & 13,5 \\
\hline Maschinenbau & 24.625 & 52,9 & 167.486 & 76,6 & 16.828 & 36,1 & 40.345 & 7,1 & 2.903 & 6,2 & 3.829 & 0,7 & 62 & 0,1 & 17.041 & 3,0 \\
\hline Elektrotechnik & 62.071 & 71,5 & 1.122 .095 & 63,0 & 15.451 & 17,8 & 22.750 & 8,6 & 6.310 & 7,3 & 3.327 & 1,3 & 606 & 0,7 & 25.095 & 9,4 \\
\hline Metallindustrie & 160.002 & 66,2 & & 70,0 & 48.694 & 20,2 & 99.882 & 6,2 & 18.429 & 7,6 & 28.101 & 1,8 & 2.109 & 0,9 & 109.127 & 6,8 \\
\hline
\end{tabular}


Eine ähnliche Entwicklung läßt sich für die weibliche Beschäftigung feststellen, wenn man die Gruppe der Mithelfenden Familienangehörigen näher betrachtet. Im gesamten Metallsektor war die Beschäftigungsentwicklung dieser Arbeitskräftegruppe für Männer und Frauen relativ gleich: Ihre Zahl nahm von 1925 bis 1933 um gut 40 Prozent zu, der Frauenanteil innerhalb der Gruppe veränderte sich nicht (1925: 19.433 männliche und 12.935 weibliche Mithelfende Familienangehörige). Aber besonders innerhalb der Gruppe der weiblichen Beschäftigten wuchs der Anteil der Mithelfenden Familienangehörigen beträchtlich, und dies gilt besonders für die Metallwarenindustrie (Tab. 5). 1933 wurde nahezu ein Zehntel der weiblichen Beschäftigten in der Metallwarenindustrie als Mithelfende Familienangehörige registriert. Die relativ große Anzahl weiblicher Arbeitskräfte in dieser Berufsstellung läßt sich jedoch wohl kaum als Konkurrenz für männliche Arbeitskräfte interpretieren. Eher wird es sich im Zuge der Krise um ein komplementäres Verhältnis zwischen Männer- und Frauenarbeit gehandelt haben, wobei Frauen als Ehefrauen und Töchter in der Funktion der Mithelfenden Familienangehörigen in der Werkstatt des (alleintätig selbständigen) männlichen Familienoberhaupts unterstützend arbeiteten. Unter die 1933 gezählten männlichen Mithelfenden Familienangehörigen könnte ein größerer Anteil von Söhnen fallen, die in solch einem familiären Arbeitsverbund tätig waren, weil ihnen angesichts von Wirtschaftskrise und hoher Erwerbslosigkeit keine außerhäusliche Erwerbstätigkeit offenstand. Dies eindeutig zu belegen, fehlt es jedoch an einer Altersdifferenzierung der Betriebszählungsdaten.

\section{Resümee}

Im Untersuchungszeitraum von 1895 bis 1933 hat die Elektrotechnische Industrie entscheidend zur Ausweitung der Frauenarbeit in der Metallindustrie beigetragen..$^{30}$ Die Metallwarenindustrie blieb zwar relativ bis in die zwanziger Jahre hinein der wichtigste Beschäftigungsbereich für Frauen, doch die Zuwachsraten waren hier vergleichsweise minimal. Höher noch als in der Elektrotechnischen Industrie waren die Zuwachsraten dagegen in der Maschinenbauindustrie. Dies ist jedoch vor allem auf die Rekrutierung von weiblichen Angestellten zurückzuführen. ${ }^{31}$ Die Entwicklung des Frauenanteils unter den Angestellten in der Maschinenbauindustrie unterstreicht diese Feststellung: Im Jahre 1895 betrug der Frauenanteil lediglich 1,4 Prozent (189), er stieg dann über 9,8 Prozent (3.264) im Jahre 1907 auf 28,1 Prozent (26.650) im Jahre 1925. Im Vergleich zu diesen Werten veränderte sich der Frauenanteil unter den Arbeiterinnen und Arbeitern kaum von 1 Prozent (3.562) über 1,1 Prozent (7.097) auf 4,1 Prozent (41.040).

Die nach Wirtschaftsgruppen differenzierte Untersuchung der männlichen und weiblichen Beschäftigungsentwicklung belegt, daß die Elektrotechnische Industrie für die Zunahme der Frauenarbeit in der Metallindustrie von entscheidender Bedeutung war. In diesem Sektor arbeitete 1925 mehr als ein Drittel aller weiblichen Metallbeschäftigten, während die Maschi-

$30 \mathrm{Vg1.} \mathrm{D.} \mathrm{Schmidt,} \mathrm{Taylorismus} \mathrm{und} \mathrm{Geschlechterverhältnis.} \mathrm{Was} \mathrm{der} \mathrm{„Dritte} \mathrm{Mann“} \mathrm{mit} \mathrm{dem} \mathrm{„Tod} \mathrm{des}$ Gelernten" zu tun hat, in: G. Hurrle/F.-J. Jelich/J. Seitz (Hg.), Arbeit und Technik im sozialen Prozeß, Marburg 1991, S. 68-79.

31 Siehe auch $A$. Willms, Segregation auf Dauer? Zur Entwicklung des Verhältnisses von Frauenarbeit und Männerarbeit in Deutschland 1882-1980, in: Muller/Willms/Handl 1983, S. 107-182, bes. S. $149 \mathrm{f}$. Willms stellt für den Gewerbebereich insgesamt fest, daß der Zustrom von Frauen allein dem Angestelltenbereich zugekommen sei, während es unter den Arbeiterinnen lediglich eine Verschiebung hin zu neuen Arbeitsplätzen gegeben habe. 
nenbauindustrie nur annähernd zwei Fünftel aufnahm. Die bemerkenswerte Steigerung des Frauenanteils in der Elektrotechnischen Industrie von 14,3 Prozent im Jahre 1907 auf 24,8 Prozent im Jahre 1925 war in erster Linie für die Zunahme des Frauenanteils in der Metallindustrie insgesamt verantwortlich.

Die Differenzierung nach Berufsstellungen zeigt, daß etwa um die Jahrhundertwende ein Wandel in der Beschäftigtenstruktur einsetzte. Zwischen 1895 (51.704) und 1907 (113.409) führte zwar eine expansive Produktionsentwicklung noch zu einer Verdopplung der Zahl der Arbeiterinnen, ihre relative Bedeutung innerhalb der Beschäftigtenstruktur nahm jedoch schon von 85,8 Prozent auf 82,9 Prozent ab. ${ }^{32}$ Parallel dazu gewannen insbesondere die weiblichen Angestellten an Bedeutung. Vor allem in den ausgeprägtesten Männerdomänen wuchs diese Beschäftigtengruppe überproportional. Soweit aus den Betriebszählungen ablesbar, ist diese Umstrukturierung kennzeichnend für die weibliche Beschäftigungsentwicklung bis 1933. In der männlichen Beschäftigungsentwicklung tritt solch ein Strukturwandel nicht so deutlich in Erscheinung. Der Anteil der Arbeiter stieg zwischen 1895 und 1907 zunächst sogar noch von 75,4 Prozent auf 81,1 Prozent.

Der Frauenanteil an der Lohnarbeiterschaft entsprach insgesamt etwa dem Frauenanteil unter den Beschäftigten der Metallindustrie insgesamt (Tab. 2) und variierte wie dieser stark nach Wirtschaftsgruppen. Während des Ersten Weltkrieges wurde die Zehn-Prozent-Marke höchstwahrscheinlich erstmals überschritten, aber nur wenig darüber pendelte sich der Frauenanteil in der Weimarer Republik ein.

Diese Realität der Zahlen deckte sich indes nicht mit der zeitgenössischen Wahrnehmung der Frauenarbeit in der Metallindustrie. Die Wahrnehmung der geschlechtsspezifischen Beschäftigungsentwicklung war vielmehr bestimmt von dem vom Durchschnitt der Metallindustrie erheblich abweichenden Geschlechterverhältnis in einzelnen Metallbranchen. Aufgrund der heterogenen Verhältnisse im Metallsektor ist es durchaus plausibel, daß die im Einzelfall starke Präsenz von Frauen in einer Branche oder einem Betrieb die Wahrnehmung von Frauenarbeit stärker beeinflußte als die Arbeitsmarktzahlen der Reichsstatistik. Andererseits haben Frauen möglicherweise gerade in Branchen, in denen sie eher vereinzelt auftraten und damit besonders exotisch wirkten, stärkere Aufmerksamkeit auf sich gezogen.

Der heuristische Wert von Fallstudien zur geschlechtsspezifischen Arbeitsmarktentwicklung in einzelnen Industrien besteht darin, nachweisen zu können, wie grundlegend das Merkmal Geschlecht den Arbeitsmarkt segmentierte. Auch kann gezeigt werden, wie die konjunkturelle Entwicklung verschiedene Branchen auf unterschiedliche Weise erfaßt und damit eine unterschiedliche Beschäftigungsentwicklung für Männer und Frauen verursacht. Die auf eine bestimmte Industrie konzentrierte Untersuchung der geschlechtsspezifischen Arbeitsmarktverhältnisse gibt einer Fallstudie darüber hinaus eine politische Bedeutung. Politisch gesehen scheint es wenig hilfreich, ganz allgemein zu belegen, daß Frauen Männer nicht vom Arbeitsmarkt verdrängt haben. ${ }^{33}$ Im Kontext der Gewerkschaftspolitik wird sehr

32 Zu bedenken ist, daß in den ersten Betriebszählungen die Mithelfenden Familienangehörigen nicht adäquat erfaßt wurden, so daß deren Anteil vermutlich höher zu veranschlagen wäre als er aus der Statistik zu errechnen ist. Daraus folgt für die Arbeiterinnen ein wahrscheinlich relativ stärkerer Bedeutungsverlust innerhalb der Beschäftigtenstruktur.

33 Siehe C. Mockel, Zur Frage der Verdrängung männlicher durch weibliche Arbeitskräfte, untersucht auf der Basis der Struktur der Frauenerwerbsarbeit in Berlin 1871 bis 1914, in: Hefte zur Wirtschaftsgeschichte, H. 2, Berlin 1990, S. 64-79. 
deutlich, daß die geschlechtsspezifische Arbeitsmarktsegregation in einem begrenzten Industriebereich analysiert werden muß, da die Arbeiterschaft in erster Linie ihr eigenes Umfeld registrierte, das heißt den Erwerbsbereich, für den sie vorgebildet war und in dem ihre Erwerbsbiographie vorrangig verankert war.

Gerade der Gewerkschaft wäre es möglich gewesen, einen genaueren Überblick über die geschlechtsspezifische Beschäftigungsentwicklung zu gewinnen und die gewonnenen Kenntnisse weiter zu vermitteln. Eine Politik auf Basis allgemeinster Durchschnittswerte war ebenso unredlich wie eine Politik, die an die in einer einzelnen Branche oder im Betrieb gewachsenen männlichen Ressentiments gegen Frauenarbeit anknüpfte. Die Wirksamkeit einer solchen Politik zeigt sich indes nicht zuletzt in der Fortschreibung der Verdrängungsthese in der Forschung, wo dieses Klischee ebenso überlebt hat wie das Stereotyp des geringen gewerkschaftlichen Organisationsgrades von Frauen. Für eine Geschlechtergeschichte der Arbeit erweist sich die Reichsstatistik, so sie denn richtig gelesen wird, als wichtige Quelle, um gängige Topoi, die aus der Altagsverständigung in die historische Forschung übernommen worden sind, endlich zu korrigieren. 